TAIWANESE JOURNAL OF MATHEMATICS

Vol. 15, No. 2, pp. 457-472, April 2011

This paper is available online at http://www.tjm.nsysu.edu.tw/

\title{
FIXED POINT THEOREMS AND ERGODIC THEOREMS FOR NONLINEAR MAPPINGS IN HILBERT SPACES
}

\author{
Wataru Takahashi and Jen-Chih Yao*
}

\begin{abstract}
In this paper, we first consider classes of nonlinear mappings containing the class of firmly nonexpansive mappings which can be deduced from an equilibrium problem in a Hilbert space. Further, we deal with fixed point theorems and ergodic theorems for these nonlinear mappings.
\end{abstract}

\section{INTRODUCTION}

Let $H$ be a real Hilbert space and let $C$ be a nonempty closed convex subset of $H$. Then a mapping $T: C \rightarrow H$ is said to be nonexpansive if $\|T x-T y\| \leq$ $\|x-y\|$ for all $x, y \in C$. We know that if $C$ is a bounded closed convex subset of $H$ and $T: C \rightarrow C$ is nonexpansive, then the set $F(T)$ of fixed points of $T$ is nonempty. Further, from Baillon [1] we know the first nonlinear ergodic theorem in a Hilbert space: Let $C$ be a nonempty bounded closed convex subset of $H$ and let $T: C \rightarrow C$ be nonexpansive. Then, for any $x \in C$,

$$
S_{n} x=\frac{1}{n} \sum_{k=0}^{n-1} T^{k} x
$$

converges weakly to an element $z \in F(T)$. An important example of nonexpansive mappings in a Hilbert space is a firmly nonexpansive mapping. A mapping $F$ is said to be firmly nonexpansive if

$$
\|F x-F y\|^{2} \leq\langle x-y, F x-F y\rangle
$$

for all $x, y \in C$; see, for instance, Browder [3], Goebel and Kirk [5], Goebel and Reich [6], Reich and Shoikhet [11] and Takahashi [13]. It is known that a mapping $F: C \rightarrow H$ is firmly nonexpansive if and only if

$$
\|F x-F y\|^{2}+\|(I-F) x-(I-F) y\|^{2} \leq\|x-y\|^{2}
$$

Received August 10, 2009.

2000 Mathematics Subject Classification: Primary 47H10; Secondary 47H05.

Key words and phrases: Nonexpansive mapping, Nonspreading mapping, Equilibrium problem, Fixed point, Mean convergence, Hilbert space.

*Corresponding author. 
for all $x, y \in C$, where $I$ is the identity mapping on $H$. It is also known that a firmly nonexpansive mapping $F$ can be deduced from an equilibrium problem in a Hilbert space as follows: Let $C$ be a nonempty closed convex subset of $H$ and let $f: C \times C \rightarrow \mathbb{R}$ be a bifunction satisfying the following conditions:

(A1) $f(x, x)=0, \quad \forall x \in C$;

(A2) $f$ is monotone, i.e., $f(x, y)+f(y, x) \leq 0, \quad \forall x, y \in C$;

(A3) $\lim _{t \downarrow 0} f(t z+(1-t) x, y) \leq f(x, y), \quad \forall x, y, z \in C$;

(A4) for each $x \in C, y \mapsto f(x, y)$ is convex and lower semicontinuous.

We know the following lemma; see, for instance, [2] and [4].

Lemma 1.1. Let $C$ be a nonempty closed convex subset of $H$ and let $f$ be a bifunction from $C \times C$ into $\mathbb{R}$ satisfying (A1), (A2), (A3) and (A4). Then, for any $r>0$ and $x \in H$, there exists $z \in C$ such that

$$
f(z, y)+\frac{1}{r}\langle y-z, z-x\rangle \geq 0, \quad \forall y \in C .
$$

Further, if $T_{r} x=\left\{z \in C: f(z, y)+\frac{1}{r}\langle y-z, z-x\rangle \geq 0, \forall y \in C\right\}$, then the following hold:

(1) $T_{r}$ is single-valued;

(2) $T_{r}$ is firmly nonexpansive, i.e.,

$$
\left\|T_{r} x-T_{r} y\right\|^{2} \leq\left\langle T_{r} x-T_{r} y, x-y\right\rangle, \quad \forall x, y \in H .
$$

Recently, Kohsaka and Takahashi [9] introduced the following nonlinear mapping: Let $E$ be a smooth, strictly convex and reflexive Banach space, let $J$ be the duality mapping of $E$ and let $C$ be a nonempty closed convex subset of $E$. Then, a mapping $S: C \rightarrow E$ is said to be nonspreading if

$$
\phi(S x, S y)+\phi(S y, S x) \leq \phi(S x, y)+\phi(S y, x)
$$

for all $x, y \in C$, where $\phi(x, y)=\|x\|^{2}-2\langle x, J y\rangle+\|y\|^{2}$ for all $x, y \in E$. They considered such a mapping to study the resolvents of a maximal monotone operator in the Banach space. In the case when $E$ is a Hilbert space, we know that $\phi(x, y)=\|x-y\|^{2}$ for all $x, y \in E$. So, a nonspreading mapping $S$ in a Hilbert space $H$ is defined as follows:

$$
2\|S x-S y\|^{2} \leq\|S x-y\|^{2}+\|x-S y\|^{2}
$$

for all $x, y \in C$. On the other hand, Takahashi [16] found another new nonlinear mapping called a hybrid mapping which is deduced from a firmly nonexpansive mapping. 
In this paper, we first discuss classes of nonlinear mappings containing the class of firmly nonexpansive mappings which can be deduced from a firmly nonexpansive mapping in a Hilbert space. Further, we deal with fixed point theorems and ergodic theorems for these nonlinear mappings.

\section{Preliminaries}

Throughout this paper, we denote by $\mathbb{N}$ the set of positive integers and by $\mathbb{R}$ the set of real numbers. Let $H$ be a real Hilbert space with inner product $\langle\cdot, \cdot\rangle$ and norm $\|\cdot\|$, respectively. In a Hilbert space, it is known that

$$
\|\alpha x+(1-\alpha) y\|^{2}=\alpha\|x\|^{2}+(1-\alpha)\|y\|^{2}-\alpha(1-\alpha)\|x-y\|^{2}
$$

for all $x, y \in H$ and $\alpha \in \mathbb{R}$; see, for instance, [15]. Further, in a Hilbert space, we have that

$$
2\langle x-y, z-w\rangle=\|x-w\|^{2}+\|y-z\|^{2}-\|x-z\|^{2}-\|y-w\|^{2}
$$

for all $x, y, z, w \in H$. Indeed, we have that

$$
\begin{aligned}
2\langle x-y, z-w\rangle= & 2\langle x, z\rangle-2\langle x, w\rangle-2\langle y, z\rangle+2\langle y, w\rangle \\
= & \left(-\|x\|^{2}+2\langle x, z\rangle-\|z\|^{2}\right)+\left(\|x\|^{2}-2\langle x, w\rangle+\|w\|^{2}\right) \\
& +\left(\|y\|^{2}-2\langle y, z\rangle+\|z\|^{2}\right)+\left(-\|y\|^{2}+2\langle y, w\rangle-\|w\|^{2}\right) \\
= & \|x-w\|^{2}+\|y-z\|^{2}-\|x-z\|^{2}-\|y-w\|^{2} .
\end{aligned}
$$

Let $C$ be a closed convex subset of $H$ and let $T$ be a mapping of $C$ into $H$. We denote by $F(T)$ the set of all fixed points of $T$, that is, $F(T)=\{z \in C: T z=z\}$. We denote the strong convergence and the weak convergence of $\left\{x_{n}\right\}$ to $x \in H$ by $x_{n} \rightarrow x$ and $x_{n} \rightarrow x$, respectively. A mapping $T: C \rightarrow H$ is nonexpansive if

$$
\|T x-T y\| \leq\|x-y\|
$$

for all $x, y \in C$. A mapping $F: C \rightarrow H$ is firmly nonexpansive if

$$
\|F x-F y\|^{2} \leq\langle x-y, F x-F y\rangle
$$

for all $x, y \in C$. We know that a firmly nonexpansive mapping $S: C \rightarrow H$ is nonexpansive. The following lemma is in [13].

Lemma 2.1. Let $C$ be a nonempty closed convex subset of $H$ and let $f: C \rightarrow$ $(-\infty, \infty]$ be a proper convex lower semicontinuous function such that $f\left(z_{m}\right) \rightarrow \infty$ as $\left\|z_{m}\right\| \rightarrow \infty$. Then there exists an element $z_{0} \in C$ such that

$$
f\left(z_{0}\right)=\min \{f(z): z \in C\} .
$$


Let $\mathbb{N}$ be the set of positive integers and let $l^{\infty}$ be the Banach space of bounded sequences with supremum norm. Let $\mu$ be an element of $\left(l^{\infty}\right)^{*}$ (the dual space of $\left.l^{\infty}\right)$. Then, we denote by $\mu(f)$ the value of $\mu$ at $f=\left(x_{1}, x_{2}, x_{3}, \ldots\right) \in l^{\infty}$. Sometimes, we denote by $\mu_{n}\left(x_{n}\right)$ the value $\mu(f)$. A linear functional $\mu$ on $l^{\infty}$ is called a mean if $\mu(e)=\|\mu\|=1$, where $e=(1,1,1, \ldots)$. A mean $\mu$ is called a Banach limit on $l^{\infty}$ if $\mu_{n}\left(x_{n+1}\right)=\mu_{n}\left(x_{n}\right)$. We know that there exists a Banach limit on $l^{\infty}$; see [13] for more details.

\section{NONLINEAR MAPPINGS}

Let $H$ be a Hilbert space. Let $C$ be a nonempty closed convex subset of $H$ and let $T$ be a mapping of $C$ into $H$. Then, from [16], we have the following equality:

$$
\|T x-T y\|^{2}=\|x-y-(T x-T y)\|^{2}-\|x-y\|^{2}+2\langle x-y, T x-T y\rangle
$$

for all $x, y \in C$. We have also from (2) that

(4) $2\langle x-y, T x-T y\rangle=\|x-T y\|^{2}+\|y-T x\|^{2}-\|x-T x\|^{2}-\|y-T y\|^{2}$.

Further, we have that

$$
\|x-y-(T x-T y)\|^{2}=\|x-T x\|^{2}+\|y-T y\|^{2}-2\langle x-T x, y-T y\rangle .
$$

If $T: C \rightarrow H$ is firmly nonexpansive, then

$$
\|T x-T y\|^{2} \leq\langle x-y, T x-T y\rangle .
$$

So, we have from (3) that

$$
\begin{aligned}
2\|T x-T y\|^{2} & \leq 2\langle x-y, T x-T y\rangle \\
& =\|T x-T y\|^{2}-\|x-y-(T x-T y)\|^{2}+\|x-y\|^{2} \\
& \leq\|T x-T y\|^{2}+\|x-y\|^{2} .
\end{aligned}
$$

Then, we have

$$
\|T x-T y\|^{2} \leq\|x-y\|^{2}
$$

and hence

$$
\|T x-T y\| \leq\|x-y\| .
$$

Such a mapping is called a nonexpansive mapping. Thus, we can get new classes of nonlinear operators which contain the class of firmly nonexpansive mappings in 
a Hilbert space. For example, Kohsaka and Takahahi [9] obtained a nonspreading mapping, i.e.,

$$
2\|T x-T y\|^{2} \leq\|x-T y\|^{2}+\|y-T x\|^{2}
$$

for all $x, y \in C$. We know that the class of nonspreading mappings contains the class of firmly nonexpansive mappings; see [16]. From Iemoto and Takahashi [7], we know the following lemma.

Lemma 3.1. Let $C$ be a nonempty closed convex subset of $H$. Then a mapping $S: C \rightarrow H$ is nonspreading if and only if

$$
\|S x-S y\|^{2} \leq\|x-y\|^{2}+2\langle x-S x, y-S y\rangle
$$

for all $x, y \in C$.

Further, Takahashi [16] defined the following hybrid mapping, i.e.,

$$
\|T x-T y\|^{2} \leq\|x-y\|^{2}+\langle x-T x, y-T y\rangle
$$

for all $x, y \in C$. We also know that the class of hybrid mappings contains the class of firmly nonexpansive mappings; see [16]. From Takahashi [16], we know the following lemma.

Lemma 3.2. Let $H$ be a Hilbert space and let $C$ be a nonempty closed convex subset of $H$. Then a mapping $T: C \rightarrow H$ is hybrid if and only if

$$
3\|T x-T y\|^{2} \leq\|x-y\|^{2}+\|y-T x\|^{2}+\|x-T y\|^{2}
$$

for all $x, y \in C$.

So, a hybrid mapping $T: C \rightarrow H$ is different from a nonspreading mapping.

\section{Generalized Fixed Point Theorem}

In this section, we prove a generalized fixed point theorem in a Hilbert space. Before proving the theorem, we show the following lemma.

Lemma 4.1. Let $C$ be a nonempty closed convex subset of a Hilbert space $H$, let $\left\{x_{n}\right\}$ be a bounded sequence in $H$ and let $\mu$ be a Banach limit. If $g: C \rightarrow \mathbb{R}$ is defined by

$$
g(z)=\mu_{n}\left\|x_{n}-z\right\|^{2}, \quad \forall z \in C,
$$

then there exists a unique $z_{0} \in C$ such that

$$
g\left(z_{0}\right)=\min \{g(z): z \in C\} .
$$


Proof. Let $z, y \in C$ and $\alpha, \beta \in[0,1]$ with $\alpha+\beta=1$. Then, for any $n \in \mathbb{N}$ we have from (1)

$$
\left\|x_{n}-(\alpha z+\beta y)\right\|^{2} \leq \alpha\left\|x_{n}-z\right\|^{2}+\beta\left\|x_{n}-y\right\|^{2} .
$$

Since $\mu$ is a Banach limit, we have

$$
\begin{aligned}
g(\alpha z+\beta y) & =\mu_{n}\left\|x_{n}-(\alpha z+\beta y)\right\|^{2} \\
& \leq \alpha \mu_{n}\left\|x_{n}-z\right\|^{2}+\beta \mu_{n}\left\|x_{n}-y\right\|^{2} \\
& =\alpha g(z)+\beta g(y) .
\end{aligned}
$$

This implies that $g: C \rightarrow \mathbb{R}$ is a convex function. Let $z \in C$ and let $\left\{z_{m}\right\}$ be a sequence in $C$ such that $z_{m} \rightarrow z$. Then, for any $n, m \in N$ we have

$$
\begin{aligned}
\left\|x_{n}-z_{m}\right\|^{2}-\left\|x_{n}-z\right\|^{2} & \leq\left|\left\|x_{n}-z_{m}\right\|-\left\|x_{n}-z\right\|\right|\left(\left\|x_{n}-z_{m}\right\|+\left\|x_{n}-z\right\|\right) \\
& \leq M_{1}\left\|z_{m}-z\right\|,
\end{aligned}
$$

where $M_{1}=\sup _{n, m \in N}\left(\left\|x_{n}-z_{m}\right\|+\left\|x_{n}-z\right\|\right)$. So, we have

$$
g\left(z_{m}\right)-g(z) \leq M_{1}\left\|z_{m}-z\right\|
$$

Similarly, we have

$$
g(z)-g\left(z_{m}\right) \leq M_{1}\left\|z_{m}-z\right\|
$$

Therefore, we have

$$
\left|g\left(z_{m}\right)-g(z)\right| \leq M_{1}\left\|z_{m}-z\right\| .
$$

This implies that $g: C \rightarrow \mathbb{R}$ is a continuous function. Suppose that $\left\{z_{m}\right\}$ is a sequence in $C$ such that $\left\|z_{m}\right\| \rightarrow \infty$. Then, we have

$$
\begin{aligned}
\left\|z_{m}\right\|^{2} & =\left\|z_{m}-x_{n}+x_{n}\right\|^{2} \\
& =\left\|z_{m}-x_{n}\right\|^{2}+\left\|x_{n}\right\|^{2}+2\left\langle z_{m}-x_{n}, x_{n}\right\rangle \\
& \leq\left\|z_{m}-x_{n}\right\|^{2}+\left\|x_{n}\right\|^{2}+2\left(\left\|z_{m}\right\|+\left\|x_{n}\right\|\right)\left\|x_{n}\right\| \\
& \leq\left\|z_{m}-x_{n}\right\|^{2}+M_{2}^{2}+2\left(\left\|z_{m}\right\|+M_{2}\right) M_{2},
\end{aligned}
$$

where $M_{2}=\sup _{n \in \mathbb{N}}\left\|x_{n}\right\|$. Hence, we have

$$
\left\|z_{m}\right\|\left(\left\|z_{m}\right\|-2 M_{2}\right)-3 M_{2}^{2} \leq\left\|z_{m}-x_{n}\right\|^{2} .
$$

So, we have

$$
\left\|z_{m}\right\|\left(\left\|z_{m}\right\|-2 M_{2}\right)-3 M_{2}^{2} \leq \mu_{n}\left\|z_{m}-x_{n}\right\|^{2} .
$$


This implies that $g\left(z_{m}\right) \rightarrow \infty$ as $\left\|z_{m}\right\| \rightarrow \infty$. Therefore, we have from Lemma 2.1 that there exists an element $z_{0} \in C$ such that

$$
g\left(z_{0}\right)=\min \{g(z): z \in C\} .
$$

Let $z_{0}$ and $z_{1}$ be elements in $C$ such that $z_{0} \neq z_{1}$ and

$$
g\left(z_{0}\right)=g\left(z_{1}\right)=\min \{g(z): z \in C\}=r .
$$

From (1), we have

$$
\left\|x_{n}-\left(\frac{1}{2} z_{0}+\frac{1}{2} z_{1}\right)\right\|^{2}=\frac{1}{2}\left\|x_{n}-z_{0}\right\|^{2}+\frac{1}{2}\left\|x_{n}-z_{1}\right\|^{2}-\frac{1}{4}\left\|z_{0}-z_{1}\right\|^{2} .
$$

So, using $\mu$, we have

$$
\begin{aligned}
g\left(\frac{1}{2} z_{0}+\frac{1}{2} z_{1}\right) & =\frac{1}{2} g\left(z_{0}\right)+\frac{1}{2} g\left(z_{1}\right)-\frac{1}{4}\left\|z_{0}-z_{1}\right\|^{2} \\
& =r-\frac{1}{4}\left\|z_{0}-z_{1}\right\|^{2} .
\end{aligned}
$$

This is a contradiction. So, we have $z_{0}=z_{1}$.

Theorem 4.1. Let $H$ be a Hilbert space, let $C$ be a nonempty closed convex subset of $H$ and let $T$ be a mapping of $C$ into itself. Suppose that there exists an element $x \in C$ such that $\left\{T^{n} x\right\}$ is bounded and

$$
\mu_{n}\left\|T^{n} x-T y\right\|^{2} \leq \mu_{n}\left\|T^{n} x-y\right\|^{2}, \quad \forall y \in C
$$

for some Banach limit $\mu$. Then, $T$ has a fixed point in $C$.

Proof. Using a Banach limit $\mu$ on $l^{\infty}$, we can define $g: C \rightarrow \mathbb{R}$ as follows:

$$
g(z)=\mu_{n}\left\|T^{n} x-z\right\|^{2}, \quad \forall z \in C .
$$

From Lemma 4.1, there exists a unique $z_{0} \in C$ such that

$$
g\left(z_{0}\right)=\min \{g(z): z \in C\} .
$$

So, we have

$$
g\left(T z_{0}\right)=\mu_{n}\left\|T^{n} x-T z_{0}\right\|^{2} \leq \mu_{n}\left\|T^{n} x-z_{0}\right\|^{2}=g\left(z_{0}\right) .
$$

Since $T z_{0}$ is in $C$ and $z_{0} \in C$ is a unique element such that

$$
g\left(z_{0}\right)=\min \{g(z): z \in C\},
$$

we have $T z_{0}=z_{0}$. This completes the proof. 


\section{Some FiXed Point Theorems}

In this section, we obtain some fixed point theorems by using Theorem 4.1. The following is the well-known fixed point theorem for nonexpansive mappings in a Hilbert space; see, for instance, [15].

Theorem 5.1. Let $H$ be a Hilbert space and let $C$ be a nonempty closed convex subset of $H$. Let $T: C \rightarrow C$ be a nonexpansive mapping, i.e.,

$$
\|T x-T y\| \leq\|x-y\|, \quad \forall x, y \in C .
$$

Suppose that there exists an element $x \in C$ such that $\left\{T^{n} x\right\}$ is bounded. Then, $T$ has a fixed point in $C$.

Proof. Let $\mu$ be a Banach limit on $l^{\infty}$. For any $n \in \mathbb{N}$ and $y \in C$, we have

$$
\left\|T^{n+1} x-T y\right\|^{2} \leq\left\|T^{n} x-y\right\|^{2} .
$$

So, we have

$$
\mu_{n}\left\|T^{n} x-T y\right\|^{2}=\mu_{n}\left\|T^{n+1} x-T y\right\|^{2} \leq \mu_{n}\left\|T^{n} x-y\right\|^{2}
$$

for all $y \in C$. By Theorem 4.1, $T$ has a fixed point in $C$.

The following is a fixed point theorem for nonspreading mappings in a Hilbert space.

Theorem 5.2. ([9]). Let $H$ be a Hilbert space and let $C$ be a nonempty closed convex subset of $H$. Let $T: C \rightarrow C$ be a nonspreading mapping, i.e.,

$$
2\|T x-T y\|^{2} \leq\|T x-y\|^{2}+\|T y-x\|^{2}, \quad \forall x, y \in C .
$$

Suppose that there exists an element $x \in C$ such that $\left\{T^{n} x\right\}$ is bounded. Then, $T$ has a fixed point in $C$.

Proof. Let $\mu$ be a Banach limit on $l^{\infty}$. For any $n \in \mathbb{N}$ and $y \in C$, we have

$$
2\left\|T^{n+1} x-T y\right\|^{2} \leq\left\|T^{n+1} x-y\right\|^{2}+\left\|T^{n} x-T y\right\|^{2} .
$$

So, we have

$$
\begin{aligned}
2 \mu_{n}\left\|T^{n} x-T y\right\|^{2} & =2 \mu_{n}\left\|T^{n+1} x-T y\right\|^{2} \\
& \leq \mu_{n}\left\|T^{n+1} x-y\right\|^{2}+\mu_{n}\left\|T^{n} x-T y\right\|^{2} \\
& =\mu_{n}\left\|T^{n} x-y\right\|^{2}+\mu_{n}\left\|T^{n} x-T y\right\|^{2}
\end{aligned}
$$


and hence

$$
\mu_{n}\left\|T^{n} x-T y\right\|^{2} \leq \mu_{n}\left\|T^{n} x-y\right\|^{2} .
$$

By Theorem 4.1, $T$ has a fixed point in $C$.

The following is a fixed point theorem for hybrid mappings in a Hilbert space.

Theorem 5.3. ([16]). Let $H$ be a Hilbert space and let $C$ be a nonempty closed convex subset of $H$. Let $T: C \rightarrow C$ be a hybrid mapping, i.e.,

$$
\|T x-T y\|^{2} \leq\|x-y\|^{2}+\langle x-T x, y-T y\rangle, \quad \forall x, y \in C .
$$

Suppose that there exists an element $x \in C$ such that $\left\{T^{n} x\right\}$ is bounded. Then, $T$ has a fixed point in $C$.

Proof. Let $\mu$ be a Banach limit on $l^{\infty}$. We know from Lemma 3.2 that a mapping $T: C \rightarrow C$ is hybrid if and only if

$$
3\|T x-T y\|^{2} \leq\|x-y\|^{2}+\|T x-y\|^{2}+\|T y-x\|^{2}, \quad \forall x, y \in C .
$$

So, for any $n \in \mathbb{N}$ and $y \in C$, we have

$$
3\left\|T^{n+1} x-T y\right\|^{2} \leq\left\|T^{n} x-y\right\|^{2}+\left\|T^{n+1} x-y\right\|^{2}+\left\|T^{n} x-T y\right\|^{2} .
$$

So, we have

$$
\begin{aligned}
3 \mu_{n}\left\|T^{n} x-T y\right\|^{2} & =3 \mu_{n}\left\|T^{n+1} x-T y\right\|^{2} \\
& \leq 2 \mu_{n}\left\|T^{n} x-y\right\|^{2}+\mu_{n}\left\|T^{n} x-T y\right\|^{2}
\end{aligned}
$$

and hence

$$
\mu_{n}\left\|T^{n} x-T y\right\|^{2} \leq \mu_{n}\left\|T^{n} x-y\right\|^{2} .
$$

By Theorem 4.1, $T$ has a fixed point in $C$.

We can also prove the following fixed point theorem in a Hilbert space.

Theorem 5.4. Let $H$ be a Hilbert space and let $C$ be a nonempty closed convex subset of $H$. Let $T: C \rightarrow C$ be a mapping such that

$$
2\|T x-T y\|^{2} \leq\|x-y\|^{2}+\|T x-y\|^{2}, \quad \forall x, y \in C .
$$

Suppose that there exists an element $x \in C$ such that $\left\{T^{n} x\right\}$ is bounded. Then, $T$ has a fixed point in $C$. 
Proof. Let $\mu$ be a Banach limit on $l^{\infty}$. For any $n \in \mathbb{N}$ and $y \in C$, we have

$$
2\left\|T^{n+1} x-T y\right\|^{2} \leq\left\|T^{n} x-y\right\|^{2}+\left\|T^{n+1} x-y\right\|^{2} .
$$

So, we have

$$
\begin{aligned}
2 \mu_{n}\left\|T^{n} x-T y\right\|^{2} & =2 \mu_{n}\left\|T^{n+1} x-T y\right\|^{2} \\
& \leq 2 \mu_{n}\left\|T^{n} x-y\right\|^{2}
\end{aligned}
$$

and hence

$$
\mu_{n}\left\|T^{n} x-T y\right\|^{2} \leq \mu_{n}\left\|T^{n} x-y\right\|^{2} .
$$

By Theorem 4.1, $T$ has a fixed point in $C$.

We can also discuss the demiclosedness of our nonlinear mappings in a Hilbert space. The following result is well known; see [13].

Theorem 5.5. Let $H$ be a Hilbert space and let $C$ be a nonempty closed convex subset of $H$. Let $T$ be a nonexpansive mapping of $C$ into itself. Then $T$ is demiclosed, i.e., $x_{n} \rightarrow u$ and $x_{n}-T x_{n} \rightarrow 0$ imply $u \in F(T)$.

The following result is in [7].

Theorem 5.6. Let $H$ be a Hilbert space and let $C$ be a nonempty closed convex subset of $H$. Let $T$ be a nonspreadind mapping of $C$ into itself. Then $T$ is demiclosed, i.e., $x_{n} \rightarrow u$ and $x_{n}-T x_{n} \rightarrow 0$ imply $u \in F(T)$.

From Takahashi [16], we also know the following result.

Theorem 5.7. Let $H$ be a Hilbert space and let $C$ be a nonempty closed convex subset of $H$. Let $T$ be a hybrid mapping of $C$ into itself. Then $T$ is demiclosed, i.e., $x_{n} \rightarrow u$ and $x_{n}-T x_{n} \rightarrow 0$ imply $u \in F(T)$.

We can further prove the following result.

Theorem 5.8. Let $H$ be a Hilbert space and let $C$ be a nonempty closed convex subset of $H$. Let $T: C \rightarrow C$ be a mapping such that

$$
2\|T x-T y\|^{2} \leq\|x-y\|^{2}+\|T x-y\|^{2}, \quad \forall x, y \in C .
$$

Then $T$ is demiclosed, i.e., $x_{n} \rightarrow u$ and $x_{n}-T x_{n} \rightarrow 0$ imply $u \in F(T)$.

Proof. Let $\left\{x_{n}\right\} \subset C$ be a sequence such that $x_{n} \rightarrow u$ and $x_{n}-T x_{n} \rightarrow 0$ as $n \rightarrow \infty$. Then the sequences $\left\{x_{n}\right\}$ and $\left\{T x_{n}\right\}$ are bounded. Suppose that $u \neq T u$. From Opial's theorem [10], we have 


$$
\begin{aligned}
\liminf _{n \rightarrow \infty}\left\|x_{n}-u\right\|^{2} & <\liminf _{n \rightarrow \infty}\left\|x_{n}-T u\right\|^{2} \\
& =\liminf _{n \rightarrow \infty}\left\|x_{n}-T x_{n}+T x_{n}-T u\right\|^{2} \\
& =\liminf _{n \rightarrow \infty}\left\|T x_{n}-T u\right\|^{2} \\
& \leq \liminf _{n \rightarrow \infty} \frac{1}{2}\left(\left\|x_{n}-u\right\|^{2}+\left\|T x_{n}-u\right\|^{2}\right) \\
& =\liminf _{n \rightarrow \infty} \frac{1}{2}\left(\left\|x_{n}-u\right\|^{2}+\left\|T x_{n}-x_{n}+x_{n}-u\right\|^{2}\right) \\
& =\liminf _{n \rightarrow \infty}\left\|x_{n}-u\right\|^{2} .
\end{aligned}
$$

This is a contradiction. Hence we get the conclusion.

\section{Nonlinear ERdodic THEOREMS}

Baillon [1] proved the first nonlinear ergodic theorem for nonexpansive mappings in a Hilbert space.

Theorem 6.1. Let $H$ be a Hilbert space, let $C$ be a nonempty closed convex subset of $H$ and let $T$ be a nonexpansive mapping of $C$ into itself such that $F(T)$ is nonempty. Then, for any $x \in C$,

$$
S_{n} x=\frac{1}{n} \sum_{k=0}^{n-1} T^{k} x
$$

converges weakly to an element $z \in F(T)$.

We can also prove the following nonlinear ergodic theorem for our nonlinear operators in a Hilbert space.

Theorem 6.2. Let $H$ be a Hilbert space, let $C$ be a nonempty closed convex subset of $H$ and let $T$ be a mapping of $C$ into itself such that $F(T)$ is nonempty. Suppose that $T$ satisfies one of the following conditions:

(i) $T$ is nonspreading;

(ii) $T$ is hybrid;

(iii) $2\|T x-T y\|^{2} \leq\|x-y\|^{2}+\|T x-y\|^{2}, \quad \forall x, y \in C$.

Then, for any $x \in C$,

$$
S_{n} x=\frac{1}{n} \sum_{k=0}^{n-1} T^{k} x
$$

converges weakly to an element $z \in F(T)$. 
Proof. Let us prove the case of (i). We first show that $F(T)$ is closed and convex. It follows from Theorem 5.6 that $F(T)$ is closed. In fact, let $\left\{x_{n}\right\} \subset F(T)$ and $x_{n} \rightarrow z$. Then, we have $x_{n} \rightarrow z$ and $x_{n}-T x_{n}=0$. So, from Theorem 5.6 we have $z=T z$. Let us show that $F(T)$ is convex. Let $x, y \in F(T)$ and $\alpha \in[0,1]$ and put $z=\alpha x+(1-\alpha) y$. Then, we have from (1) that

$$
\begin{aligned}
\|z-T z\|^{2} & =\|\alpha x+(1-\alpha) y-T z\|^{2} \\
& =\alpha\|x-T z\|^{2}+(1-\alpha)\|y-T z\|^{2}-\alpha(1-\alpha)\|x-y\|^{2} \\
& =\alpha\|T x-T z\|^{2}+(1-\alpha)\|T y-T z\|^{2}-\alpha(1-\alpha)\|x-y\|^{2} \\
& \leq \alpha(1-\alpha)^{2}\|x-y\|^{2}+(1-\alpha) \alpha^{2}\|x-y\|^{2}-\alpha(1-\alpha)\|x-y\|^{2} \\
& =\alpha(1-\alpha)(1-\alpha+\alpha-1)\|x-y\|^{2} \\
& =0 .
\end{aligned}
$$

So, we have $T z=z$. Let $x \in C$ and let $P$ be the metric projection of $H$ onto $F(T)$. Then, we have

$$
\begin{aligned}
\left\|P T^{n} x-T^{n} x\right\| & \leq\left\|P T^{n-1} x-T^{n} x\right\| \\
& =\left\|T P T^{n-1} x-T^{n} x\right\| \\
& \leq\left\|P T^{n-1} x-T^{n-1} x\right\| .
\end{aligned}
$$

This implies that $\left\{\left\|P T^{n} x-T^{n} x\right\|\right\}$ is nonincreasing. We also know that for any $v \in C$ and $u \in F(T)$,

$$
\langle v-P v, P v-u\rangle \geq 0
$$

and hence

$$
\|v-P v\|^{2} \leq\langle v-P v, v-u\rangle .
$$

So, we get

$$
\begin{aligned}
\|P v-u\|^{2} & =\|P v-v+v-u\|^{2} \\
& =\|P v-v\|^{2}-2\langle P v-v, u-v\rangle+\|v-u\|^{2} \\
& \leq\|v-u\|^{2}-\|P v-v\|^{2} .
\end{aligned}
$$

Let $m, n \in \mathbb{N}$ with $m \geq n$. Putting $v=T^{m} x$ and $u=P T^{n} x$, we have

$$
\begin{aligned}
& \left\|P T^{m} x-P T^{n} x\right\|^{2} \\
\leq & \left\|T^{m} x-P T^{n} x\right\|^{2}-\left\|P T^{m} x-T^{m} x\right\|^{2} \\
\leq & \left\|T^{n} x-P T^{n} x\right\|^{2}-\left\|P T^{m} x-T^{m} x\right\|^{2} .
\end{aligned}
$$


So, $\left\{P T^{n} x\right\}$ is a Cauchy sequence. Since $F(T)$ is closed, $\left\{P T^{n} x\right\}$ converges strongly to an element $p$ of $F(T)$. Take $u \in F(T)$. Then we obtain, for any $n \in \mathbb{N}$,

$$
\left\|S_{n} x-u\right\| \leq \frac{1}{n} \sum_{k=0}^{n-1}\left\|T^{k} x-u\right\| \leq\|x-u\| .
$$

So, $\left\{S_{n} x\right\}$ is bounded and hence there exists a weakly convergent subsequence $\left\{S_{n_{i}} x\right\}$ of $\left\{S_{n} x\right\}$. If $S_{n_{i}} x \rightarrow v$, then we have $v \in F(T)$. In fact, for any $y \in C$ and $k \in \mathbb{N} \cup\{0\}$, we have that

$$
\begin{aligned}
\left\|T^{k+1} x-T y\right\|^{2} \leq & \left\|T^{k} x-y\right\|^{2}+2\left\langle T^{k} x-T^{k+1} x, y-T y\right\rangle \\
= & \left\|T^{k} x-y\right\|^{2}+\left\|T^{k} x-T y\right\|^{2}+\left\|T^{k+1} x-y\right\|^{2} \\
& -\left\|T^{k} x-y\right\|^{2}-\left\|T^{k+1} x-T y\right\|^{2} \\
= & \left\|T^{k} x-T y\right\|^{2}+2\left\langle T^{k} x-T y, T y-y\right\rangle+\|T y-y\|^{2} \\
& +\left\|T^{k} x-T y\right\|^{2}+\left\|T^{k+1} x-y\right\|^{2}-\left\|T^{k} x-y\right\|^{2}-\left\|T^{k+1} x-T y\right\|^{2} .
\end{aligned}
$$

So, we obtain that

$$
\begin{aligned}
2\left\|T^{k+1} x-T y\right\|^{2} \leq & 2\left\|T^{k} x-T y\right\|^{2}+2\left\langle T^{k} x-T y, T y-y\right\rangle \\
& +\|T y-y\|^{2}+\left\|T^{k+1} x-y\right\|^{2}-\left\|T^{k} x-y\right\|^{2} .
\end{aligned}
$$

Summing these inequalities with respect to $k=0,1, \ldots, n-1$, we have

$$
\begin{aligned}
2\left\|T^{n} x-T y\right\|^{2} \leq & 2\|x-T y\|^{2}+2\left\langle\sum_{k=0}^{n-1} T^{k} x-n T y, T y-y\right\rangle \\
& +n\|T y-y\|^{2}+\left\|T^{n} x-y\right\|^{2}-\|x-y\|^{2} .
\end{aligned}
$$

Deviding this inequality by $n$, we have

$$
\begin{aligned}
\frac{2}{n}\left\|T^{n} x-T y\right\|^{2} \leq & \frac{2}{n}\|x-T y\|^{2}+2\left\langle S_{n} x-T y, T y-y\right\rangle \\
& +\|T y-y\|^{2}+\frac{1}{n}\left\|T^{n} x-y\right\|^{2}-\frac{1}{n}\|x-y\|^{2},
\end{aligned}
$$

where $S_{n} x=\frac{1}{n} \sum_{k=0}^{n-1} T^{k} x$. Replacing $n$ by $n_{i}$ and letting $n_{i} \rightarrow \infty$, we obtain from $S_{n_{i}} x \rightarrow v$ that

$$
0 \leq\|T y-y\|^{2}+2\langle v-T y, T y-y\rangle .
$$

Putting $y=v$, we have

$$
0 \leq\|T v-v\|^{2}+2\langle v-T v, T v-v\rangle .
$$


So, we have $0 \leq-\|T v-v\|^{2}$ and hence $T v=v$. To complete the proof of (i), it is sufficient to show that if $S_{n_{i}} x \rightarrow v$, then $v=p$. We have, for any $u \in F(T)$,

$$
\left\langle T^{k} x-P T^{k} x, P T^{k} x-u\right\rangle \geq 0 .
$$

Since $\left\{\left\|T^{k} x-P T^{k} x\right\|\right\}$ is nonincreasing, we have

$$
\begin{aligned}
\left\langle u-p, T^{k} x-P T^{k} x\right\rangle & \leq\left\langle P T^{k} x-p, T^{k} x-P T^{k} x\right\rangle \\
& \leq\left\|P T^{k} x-p\right\| \cdot\left\|T^{k} x-P T^{k} x\right\| \\
& \leq\left\|P T^{k} x-p\right\| \cdot\|x-P x\| .
\end{aligned}
$$

Adding these inequalities from $k=0$ to $k=n-1$ and dividing $n$, we have

$$
\left\langle u-p, S_{n} x-\frac{1}{n} \sum_{k=0}^{n-1} P T^{k} x\right\rangle \leq \frac{\|x-P x\|}{n} \sum_{k=0}^{n-1}\left\|P T^{k} x-p\right\| .
$$

Since $S_{n_{i}} x \rightarrow v$ and $P T^{k} x \rightarrow p$, we have

$$
\langle u-p, v-p\rangle \leq 0 \text {. }
$$

We know $v \in F(T)$. So, putting $u=v$, we have $\langle v-p, v-p\rangle \leq 0$ and hence $\|v-p\|^{2} \leq 0$. So, we obtain $v=p$. This completes the proof of (i).

Let us prove the case of (ii). It follows from Theorem 5.7 that $F(T)$ is closed. As in the proof of (i), we can show that $F(T)$ is convex. Let $x \in C$ and let $P$ be the metric projection of $H$ onto $F(T)$. Then, as in the proof of (i), we can have that $\left\{P T^{n} x\right\}$ is a Cauchy sequence. Since $F(T)$ is closed, $\left\{P T^{n} x\right\}$ converges strongly to an element $p$ of $F(T)$. Take $u \in F(T)$. Then we obtain, for any $n \in \mathbb{N}$,

$$
\left\|S_{n} x-u\right\| \leq \frac{1}{n} \sum_{k=0}^{n-1}\left\|T^{k} x-u\right\| \leq\|x-u\| .
$$

So, $\left\{S_{n} x\right\}$ is bounded and hence there exists a weakly convergent subsequence $\left\{S_{n_{i}} x\right\}$ of $\left\{S_{n} x\right\}$. If $S_{n_{i}} x \rightarrow v$, then we have $v \in F(T)$. In fact, for any $y \in C$ and $k \in \mathbb{N} \cup\{0\}$, we have that

$$
\begin{aligned}
2\left\|T^{k+1} x-T y\right\|^{2} \leq & 2\left\|T^{k} x-y\right\|^{2}+2\left\langle T^{k} x-T^{k+1} x, y-T y\right\rangle \\
= & 2\left\|T^{k} x-y\right\|^{2}+\left\|T^{k} x-T y\right\|^{2}+\left\|T^{k+1} x-y\right\|^{2} \\
& -\left\|T^{k} x-y\right\|^{2}-\left\|T^{k+1} x-T y\right\|^{2} \\
= & 2\left\|T^{k} x-T y\right\|^{2}+4\left\langle T^{k} x-T y, T y-y\right\rangle+2\|T y-y\|^{2} \\
& +\left\|T^{k} x-T y\right\|^{2}+\left\|T^{k+1} x-y\right\|^{2}-\left\|T^{k} x-y\right\|^{2}-\left\|T^{k+1} x-T y\right\|^{2} .
\end{aligned}
$$

So, we obtain that 


$$
\begin{aligned}
3\left\|T^{k+1} x-T y\right\|^{2} \leq & 3\left\|T^{k} x-T y\right\|^{2}+4\left\langle T^{k} x-T y, T y-y\right\rangle \\
& +2\|T y-y\|^{2}+\left\|T^{k+1} x-y\right\|^{2}-\left\|T^{k} x-y\right\|^{2} .
\end{aligned}
$$

Summing these inequalities with respect to $k=0,1, \ldots, n-1$, we have

$$
\begin{aligned}
3\left\|T^{n} x-T y\right\|^{2} \leq & 3\|x-T y\|^{2}+4\left\langle\left\langle\sum_{k=0}^{n-1} T^{k} x-n T y, T y-y\right\rangle\right. \\
& +2 n\|T y-y\|^{2}+\left\|T^{n} x-y\right\|^{2}-\|x-y\|^{2} .
\end{aligned}
$$

Deviding this inequality by $n$, we have

$$
\begin{aligned}
\frac{3}{n}\left\|T^{n} x-T y\right\|^{2} \leq & \frac{3}{n}\|x-T y\|^{2}+4\left\langle S_{n} x-T y, T y-y\right\rangle \\
& +2\|T y-y\|^{2}+\frac{1}{n}\left\|T^{n} x-y\right\|^{2}-\frac{1}{n}\|x-y\|^{2},
\end{aligned}
$$

where $S_{n} x=\frac{1}{n} \sum_{k=0}^{n-1} T^{k} x$. Replacing $n$ by $n_{i}$ and letting $n_{i} \rightarrow \infty$, we obtain from $S_{n_{i}} x \rightarrow v$ that

$$
0 \leq 2\|T y-y\|^{2}+4\langle v-T y, T y-y\rangle .
$$

Putting $y=v$, we have

$$
0 \leq 2\|T v-v\|^{2}+4\langle v-T v, T v-v\rangle .
$$

So, we have $0 \leq-2\|T v-v\|^{2}$ and hence $T v=v$. To complete the proof of (ii), it is sufficient to show that if $S_{n_{i}} x \rightarrow v$, then $v=p$. As in the proof of (i), we can prove $v=p$. This completes the proof of (ii).

As in the proofs of (i) and (ii), we can prove the case of (iii).

\section{ACKNOWLEDGMENTS}

The first author and the second author were partially supported by Grant-in-Aid for Scientific Research No. 19540167 from Japan Society for the Promotion of Science and by the grant NSC 98-2115-M-110-001, respectively.

\section{REFERENCES}

1. J.-B. Baillon, Un theoreme de type ergodique pour les contractions non lineaires dans un espace de Hilbert, C. R. Acad. Sci. Paris Ser. A-B, 280 (1975), 1511-1514.

2. E. Blum and W. Oettli, From optimization and variational inequalities to equilibrium problems, Math. Student, 63 (1994), 123-145.

3. F. E. Browder, Convergence theorems for sequences of nonlinear operators in Banach spaces, Math. Z., 100 (1967), 201-225. 
4. P. L. Combettes and A. Hirstoaga, Equilibrium problems in Hilbert spaces, J. Nonlinear Convex Anal., 6 (2005), 117-136.

5. K. Goebel and W. A. Kirk, Topics in metric fixed point theory, Cambridge University Press, Cambridge, 1990.

6. K. Goebel and S. Reich, Uniform convexity, hyperbolic geometry, and nonexpansive mappings, Marcel Dekker Inc., New York, 1984.

7. S. Iemoto and W. Takahashi, Approximating fixed points of nonexpansive mappings and nonspreading mappings in a Hilbert space, Nonlinear Anal., 71 (2009), 20822089.

8. F. Kohsaka and W. Takahashi, Existence and approximation of fixed points of firmly nonexpansive-type mappings in Banach spaces, SIAM. J. Optim., 19 (2008), 824-835.

9. F. Kohsaka and W. Takahashi, Fixed point theorems for a class of nonlinear mappings related to maximal monotone operators in Banach spaces, Arch. Math., 91 (2008), 166-177.

10. Z. Opial, Weak convergence of the sequence of successive approximations for nonexpansive mappings, Bull. Amer. Math. Soc., 73 (1967), 591-597.

11. S. Reich and D. Shoikhet, Nonlinear semigroups, fixed points, and geometry of domains in Banach spaces, Imperial College Press, London, 2005.

12. W. Takahashi, A nonlinear ergodic theorem for an amenable semigroup of nonexpansive mappings in a Hilbert space, Proc. Amer. Math. Soc., 81 (1981), 253-256.

13. W. Takahashi, Nonlinear Functional Analysis. Fixed Point Theory and its Applications, Yokohama Publishers, Yokohama, 2000.

14. W. Takahashi, Convex Analysis and Approximation of Fixed Points, Yokohama Publishers, Yokohama, 2000, (in Japanese).

15. W. Takahashi, Introduction to Nonlinear and Convex Analysis, Yokohama Publishers, Yokohama, 2005, (in Japanese).

16. W. Takahashi, Fixed point theorems for new nonlinear mappings in a Hilbert space, J. Nonlinear Convex Anal., 11 (2010), 78-88.

\author{
Wataru Takahashi \\ Department of Applied Mathematics \\ National Sun Yat-sen University \\ Kaohsiung 80424, Taiwan \\ E-mail: wataru@is.titech.ac.jp \\ Jen-Chih Yao \\ Center for General Education \\ Kaohsiung Medical University \\ Kaohsiung 80707, Taiwan \\ E-mail: yaojc@math.nsysu.edu.tw
}

